\title{
Screening for Osteomyelitis Using Thermography in Patients with Diabetic Foot
}

\author{
Makoto Oe, ${ }^{1}$ Rie Roselyne Yotsu, ${ }^{1,2}$ Hiromi Sanada, ${ }^{1}$ \\ Takashi Nagase, ${ }^{1}$ and Takeshi Tamaki ${ }^{2}$ \\ ${ }^{1}$ Department of Gerontological Nursing/Wound Care Management, Graduate School of Medicine, University of Tokyo, \\ Tokyo 113-0033, Japan \\ ${ }^{2}$ Department of Dermatology, National Center for Global Health and Medicine (NCGM) Hospital, 1-21-1 Toyama, Shinjuku-ku, \\ Tokyo 162-8655, Japan \\ Correspondence should be addressed to Rie Roselyne Yotsu; yotsurie@hotmail.com
}

Received 27 September 2012; Accepted 13 March 2013

Academic Editor: Marco A. C. Frade

Copyright ( 2013 Makoto Oe et al. This is an open access article distributed under the Creative Commons Attribution License, which permits unrestricted use, distribution, and reproduction in any medium, provided the original work is properly cited.

\begin{abstract}
One of the most serious complications of diabetic foot (DF) is osteomyelitis, and early detection is important. To assess the validity of thermography to screen for osteomyelitis, we investigated thermographic findings in patients with both DF and osteomyelitis. The subjects were 18 diabetic patients with 20 occurrences of DF who visited a dermatology department at a hospital in Tokyo and underwent evaluation by magnetic resonance imaging (MRI) and thermography between June 2010 and July 2012. Osteomyelitis was identified by MRI. Thermographs were taken of the wounds and legs after bed rest of more than 15 minutes. Two wound management researchers evaluated the range of increased skin temperature. There were three types of distribution of increased skin temperature: the periwound, ankle, and knee patterns. Fisher's exact test revealed that the ankle pattern was significantly more common in the group with osteomyelitis than in the group without osteomyelitis $(P=0.011)$. The positive predictive value was $100 \%$, and the negative predictive value was $71.4 \%$. Our results suggest that an area of increased skin temperature extending to the ankle can be a sign of osteomyelitis. Thermography might therefore be useful for screening for osteomyelitis in patients with DF.
\end{abstract}

\section{Introduction}

Diabetic foot (DF) is defined as infection, ulceration, and/or destruction of deep tissue associated with neurological abnormalities and various degrees of peripheral vascular disease in the lower limb [1]. The prevalence of foot ulcers is $4 \%$ to $10 \%$ in patients with diabetes mellitus (DM) [2]. DF severely affects a patient's physical condition, long-term prognosis $[3,4]$, and quality of life $[5,6]$. Therefore, early healing of DF is important.

One of the most serious complications of DF is osteomyelitis, and its diagnosis and treatment (surgery and/or long-term antibiotics) have been long-standing controversies [7-9]. Therefore, early detection of osteomyelitis is important. Although swelling and warmth are associated with osteomyelitis, it is difficult to diagnose the presence of osteomyelitis in DF by physical examination because the plantar skin has an especially thick layer of stratum corneum. Furthermore, inflammatory pain may occasionally be overlooked in diabetic patients due to sensory disturbance. Although biopsy or magnetic resonance imaging (MRI) is the gold standard for diagnosing osteomyelitis [7-9], it is accompanied by disadvantages such as invasiveness, a high cost, and non-real-time diagnosis.

One of the possible tools for detecting inflammation or infection in DF is thermography. Several previous studies have indicated the usefulness of thermometry both for early detection of inflammation and for prevention and home monitoring of ulceration risk. Armstrong et al. [11] showed that monitoring skin temperature reduced the risk of diabetic foot ulceration. This study was further supported by multiple independent randomized controlled trials reporting similar findings and approximately 4 - to 10 -fold reductions in reulceration for patients using home-based thermometry devices $[12,13]$. Compared with a conventional device, such as the contact infrared skin thermometer (TempTouch, Xilas 
TABLE 1: Standard values used for nerve conduction velocity exam in the study.

\begin{tabular}{lccc}
\hline & Median nerve & Tibial nerve & Peroneal nerve \\
\hline M-wave amplitude $(\mathrm{mV})$ & $4-25$ & $7-40$ & - \\
Latency $(\mathrm{ms})$ & $<4.5$ & $<7.5$ & $<7.0$ \\
MCV $(\mathrm{m} / \mathrm{s})$ & $45-65$ & $40-60$ & $40-60$ \\
SNAP $(\mu \mathrm{V})$ & $10-60$ & & $5-30$ \\
SCV $(\mathrm{m} / \mathrm{s})$ & $45-58$ & & $40-60$ \\
\hline
\end{tabular}

MCV: motor nerve conduction velocity; SNAP: sensory nerve action potential; SCV: sensory nerve conduction velocity.

Medical Inc., Texas, USA) [11-13] for measuring the skin temperature of local points, we consider that thermography has an advantage in that it can visualize morphological patterns of temperature distribution [14-16]. Thermography is also noninvasive and quite easy to use by clinicians of various backgrounds. We previously reported a case study of a patient with both DF and osteomyelitis, detailing the thermographic findings [10]. A high temperature area was observed, not only in the wounds but also in the ankles. This case suggested that thermography might be useful for screening for DF with osteomyelitis.

Therefore, the purpose of this study was to investigate thermographic findings in patients with both DF and osteomyelitis in a larger number of patients and to assess the validity of screening for osteomyelitis using thermography.

\section{Methods}

This was a cross-sectional study. The subjects were patients with DF who visited a dermatology department at a hospital in Tokyo and underwent evaluation by MRI and thermography between June 2010 and July 2012.

Osteomyelitis was identified by MRI. Interpretations of diagnostic images, including MRI scans, by radiologists were collected from medical records. The criteria for diagnosing osteomyelitis were hypointense signal within the bone on T1 weighted images and hyperintense signal within the bone on T2 weighted images, in direct continuity with abnormal high signal in the surrounding soft tissues of the ulcer [17]. Thermographs were taken of the wounds and legs after bed rest of more than 15 minutes using a Thermotracer TH7800N (NEC Avio Infrared Technologies Co., Ltd., Tokyo, Japan) or a Thermo Shot F30S (NEC Avio Co., Ltd.). We adjusted temperature intervals in the thermographs at $1.5^{\circ} \mathrm{C}$ using NS9200 software (NEC Avio Co., Ltd.). Two wound management researchers evaluated the distribution of increased skin temperature. For evaluation, the investigators were blinded to data other than the thermographic images and location of the diabetic foot. Inflammation in the soft tissue was identified by MRI based on interpretations by a radiologist, and angiopathy was identified by the ankle-brachial index (ABI) and the toe-brachial index (TBI) as influential factors of skin temperature. The criteria for diagnosing angiopathy were as follows: (1) ABI no more than 0.9, or (2) ABI no less than 1.4, and TBI no more than 0.7 [18]. Evaluation of peripheral neuropathy was performed by means of nerve conduction velocity. Median, tibial, and peroneal nerves were
TABLE 2: Characteristics of subjects.

\begin{tabular}{lc}
\hline & $N=18$ \\
\hline Age, years & $66.8 \pm 15.0$ \\
$\quad$ Mex & \\
$\quad$ Female & $15(83.3)$ \\
Duration of diabetes, years & $3 .(16.7)$ \\
HbAlc $(\%)^{*}$ & $22.4 \pm 13.0$ \\
Angiopathy** & $8.4 \pm 2.6$ \\
Neuropathy ${ }^{* *}$ & $10(55.6)$ \\
Renal dialysis & $13(72.2)$ \\
Retinopathy* & $4 .(22.2)$ \\
History of myocardial infarction & $16(94.1)$ \\
\hline
\end{tabular}

Mean $\pm \mathrm{SD}, n$ (\%). ${ }^{*} n=17 .{ }^{* *}$ The criteria for diagnosing angiopathy were as follows: (1) ABI no more than 0.9, or (2) ABI no less than 1.4, and TBI no more than $0.7{ }^{* * *}$ Diagnosis of neuropathy was made according to the findings from the nerve conduction velocity exams.

tested for motor nerve conduction velocity, and median and sural nerves were tested for sensory nerve conduction velocity. Table 1 gives the standard values used in hospital for the exam. The final diagnosis was made with the neurology specialists by assessing patient's nerve conduction velocity results against these standard values. Retinopathy, renal dialysis, and history of myocardial infarction were determined using the medical records.

2.1. Statistical Analysis. Quantitative data are expressed as means \pm standard deviation. The relationships between the thermographic findings and osteomyelitis were analyzed using Fisher's exact test. To assess the validity of the thermographic findings for screening for osteomyelitis, the sensitivity, specificity, and positive and negative predictive values were calculated. Statistical analysis was performed using IBM SPSS Statistics 20 (IBM, Armonk, NY, USA). The level of statistical significance was $P=0.05$.

2.2. Ethical Considerations. This research was approved by the Ethics Committee at National Center for Global Health and Medicine Hospital. All patients gave their written informed consent.

\section{Results}

Eighteen patients with 20 occurrences of DF were included in this study (Tables 2 and 3 ). Ten occurrences of DF were 

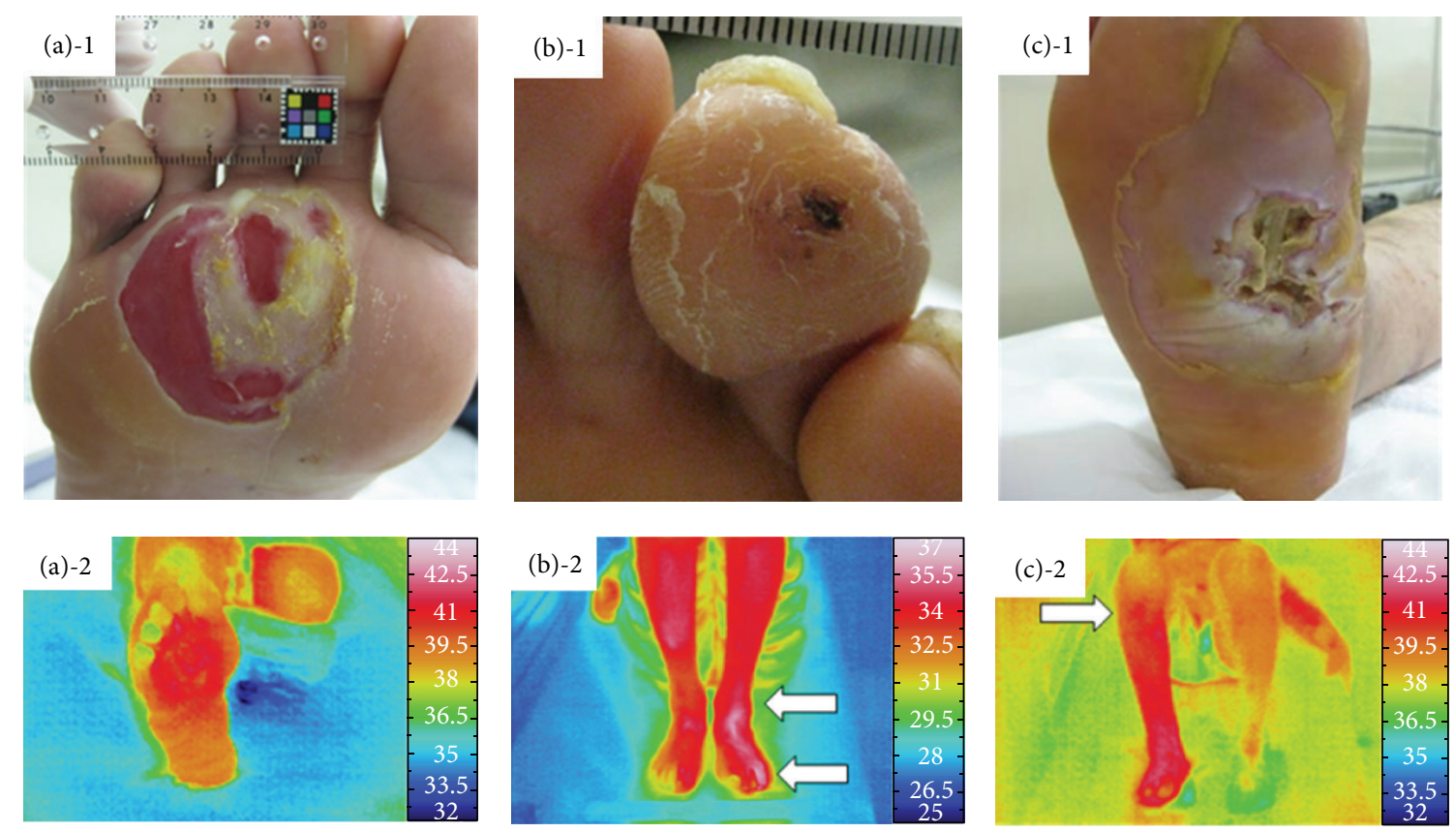

FIGURE 1: Thermographic patterns in patients with diabetic foot. (a): Periwound pattern, (a)-1: example of the periwound pattern: ulcer in the sole. (a)-2: Thermographic image of (a)-1. Distribution of increased skin temperature is found in the periwound area. (b): Ankle pattern, (b)-1: example of the ankle pattern: ulcer in the fourth toe. (b)-2: Thermographic image of (b)-1. Distribution of increased skin temperature extends from the fourth toe to the ankle (arrows). (c): Knee pattern, (c)-1: example of the knee pattern: ulcer in the sole. (c)-2: Thermographic image of (c)-1. Distribution of increased skin temperature extends to the knee (arrow). (b)-1 and (b)-2 are reproduced from [10], with permission of the Journal of Wound Care.

TABLE 3: Characteristics of diabetic foot.

\begin{tabular}{|c|c|}
\hline & $N=20$ \\
\hline \multicolumn{2}{|l|}{ Diabetic foot } \\
\hline Ulcer & $11(55.0)$ \\
\hline Gangrene & 6. $(30.0)$ \\
\hline Cellulitis & 3. $(15.0)$ \\
\hline \multicolumn{2}{|l|}{ Location of diabetic foot } \\
\hline Toe & 5. $(25.0)$ \\
\hline Sole & $12(60.0)$ \\
\hline Entire foot & 3. $(15.0)$ \\
\hline \multicolumn{2}{|l|}{ Evaluation of MRI } \\
\hline Osteomyelitis (+) inflammation of soft tissues (-) & 4. $(20.0)$ \\
\hline Osteomyelitis (-) inflammation of soft tissues $(+)$ & 6. $(30.0)$ \\
\hline Osteomyelitis $(+)$ inflammation of soft tissues $(+)$ & 6. $(30.0)$ \\
\hline Osteomyelitis (-) inflammation of soft tissues $(-)$ & 4. $(20.0)$ \\
\hline
\end{tabular}

complicated by osteomyelitis. There were three types of distribution of increased skin temperature: the periwound, ankle, and knee patterns (Figure 1). The periwound pattern indicated that increased skin temperature was observed in the periwound area. The ankle pattern indicated that the area of increased skin temperature extended to the ankle. The knee pattern indicated that the area of increased skin temperature extended to the knee. The room temperature
TABLE 4: Thermographic findings and osteomyelitis.

\begin{tabular}{|c|c|c|c|}
\hline & \multicolumn{2}{|c|}{ Osteomyelitis } & \multirow[t]{2}{*}{$P$} \\
\hline & Yes $(n=10)$ & No $(n=10)$ & \\
\hline Periwound pattern & & & 1.000 \\
\hline Yes & 0. (0.0.) & 1. (10.0.) & \\
\hline No & $10(100.0)$ & 9. (90.0.) & \\
\hline Ankle pattern & & & 0.011 \\
\hline Yes & 6. $(60.0)$. & 0. (0.0.) & \\
\hline No & 4. (40.0.) & $10(100.0)$ & \\
\hline Knee pattern & & & 0.170 \\
\hline Yes & 2. $(20.0)$. & 6. (60.0.) & \\
\hline No & 8. (80.0.) & 4. (40.0.) & \\
\hline
\end{tabular}

$n$ (\%), Fisher's exact test. Periwound pattern: increased skin temperature is observed in the periwound area. Ankle pattern: the area of increased skin temperature extends to the ankle. Knee pattern: the area of increased skin temperature extends to the knee.

when thermography was performed was controlled at $27.3 \pm$ $2.8^{\circ} \mathrm{C}$.

We compared the types of distribution of increased skin temperature between the group with osteomyelitis and the group without osteomyelitis. Fisher's exact test revealed that the ankle pattern was significantly more common in the group with osteomyelitis than in the group without osteomyelitis (Table 4). The sensitivity of the positive ankle pattern to recognize osteomyelitis was $60.0 \%$, the specificity was $100 \%$, the positive predictive value was $100 \%$, and 
TABLE 5: Thermographic findings and the site of osteomyelitis.

\begin{tabular}{lll}
\hline ID & Site of osteomyelitis & Thermographic findings \\
\hline 1 & 1st distal phalange & Ankle pattern \\
2 & 4th proximal and middle phalange & Ankle pattern \\
3 & 5th metatarsal bone & Knee pattern \\
4 & 1st proximal phalange & Ankle pattern \\
5 & 1st proximal phalange and metatarsal bone & * $^{*}$ \\
6 & 5th proximal, middle and distal phalange, and metatarsal bone & Ankle pattern \\
7 & 4th metatarsal bone & $\mathcal{-}^{*}$ \\
8 & 1st proximal, middle, and distal phalange & Ankle pattern \\
9 & 2nd proximal phalanges and metatarsal bone & Knee pattern \\
10 & 5th proximal phalange & Ankle pattern \\
\hline
\end{tabular}

${ }^{*}$ No observation of increased skin temperature.

the negative predictive value was $71.4 \%$. Table 5 shows the site of osteomyelitis and the types of distribution of increased skin temperature in each case. The site of osteomyelitis and the types of distribution of increased skin temperature seem to be unrelated, as far as we can observe from this result.

\section{Discussion}

The present study is the first to show a correlation between the ankle pattern in thermography (area of increased skin temperature extending to the ankle) and the presence of osteomyelitis in multiple DF patients. Furthermore, the positive predictive value of the ankle pattern was high, indicating the high validity of this finding. This result suggests that thermography is useful for screening for DF with osteomyelitis.

In this study, we considered that skin temperature increase detected by thermography was due to inflammation of the tissues, including the bone. Fever is one of the signs of inflammation [19]. A number of studies have previously highlighted the usefulness of thermometry in monitoring skin temperature to identify inflammation or infection of the lower extremities in patients with DM. Armstrong et al. [20] reported that there were differences in skin temperature between the affected feet and the contralateral feet in patients with Charcot's arthropathy $\left(8.3^{\circ} \mathrm{F}\right)$ and in patients with neuropathic ulcer $\left(5.6^{\circ} \mathrm{F}\right)$, with no difference identified in patients with asymptomatic sensory neuropathy. Our previous study showed that signs of inflammation were detected by thermography and ultrasonography in $10 \%$ of the calluses in the diabetic group without ulcers [21].

We designated the thermographic finding as "the ankle pattern" when the area of increased skin temperature extended to the ankle. We believe that "the ankle pattern" is a thermographic indicator for screening for osteomyelitis in DF patients, as we reported previously in a single case [10]. It is noteworthy that the specificity and the positive predictive value of the ankle pattern were $100 \%$ for the presence of osteomyelitis, indicating the surprisingly high validity of this thermographic finding. As stated above, thermography has an advantage over conventional thermometry in that the morphological patterns of temperature distribution can be obtained [14-16]. In this sense, thermography may be the best way to detect such a specific temperature distribution. In the majority of cases, osteomyelitis in the DF results from the contiguous spread of infection from the adjacent soft tissue to the bone [8]. We also speculate that skin temperature in the patients with osteomyelitis was increased due to inflammation in the adjacent deep tissues, such as the tendons. However, it is unclear in this study why the ankle pattern was specifically observed in the patients with osteomyelitis even in regions other than the ankle. Further investigation of this point might be necessary.

In the present study, there were four patients who had osteomyelitis without the ankle pattern. It was considered that angiopathy or inflammation in soft tissue influenced their skin temperature. The two patients with osteomyelitis had no area of increased skin temperature (Figure 2(a)); rather, they had severe angiopathy. It is known that the skin temperature of patients with ischemia is low [22]. Furthermore, skin temperature might be insensitive to inflammation due to insufficient blood supply [17]. The other two patients showed the knee pattern (Figure 2(b)). In these patients, inflammation of soft tissues was identified by MRI. The area of increased skin temperature due to extensive inflammation of soft tissues might mask the ankle pattern of osteomyelitis.

This study had several limitations. Morphological evaluations of thermographic patterns are quite subjective and can be easily affected by environmental conditions, expertise of the investigators, and possible bias of the patients' information. A more controlled method should be established for the collection and interpretation of thermographic data. In the present study, the diagnosis of osteomyelitis was based on MRI findings; biopsy of the bone or surrounding tissues was not able to be performed. Therefore, we cannot clearly understand the pathophysiological status underlying "the ankle pattern." We could not investigate grade of severity of angiopathy for application of this method because the sample size was small. There were no patients with Charcot arthropathy or reflex sympathetic dystrophy in the present series. A further study is needed to show if the ankle pattern would be less significant in these other conditions. This study 


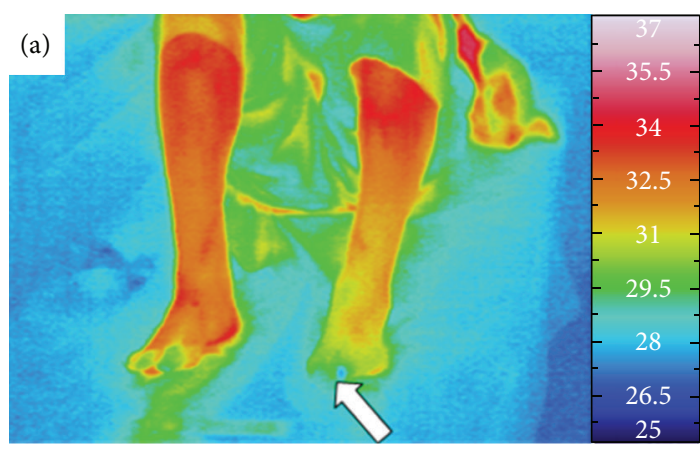

(a)

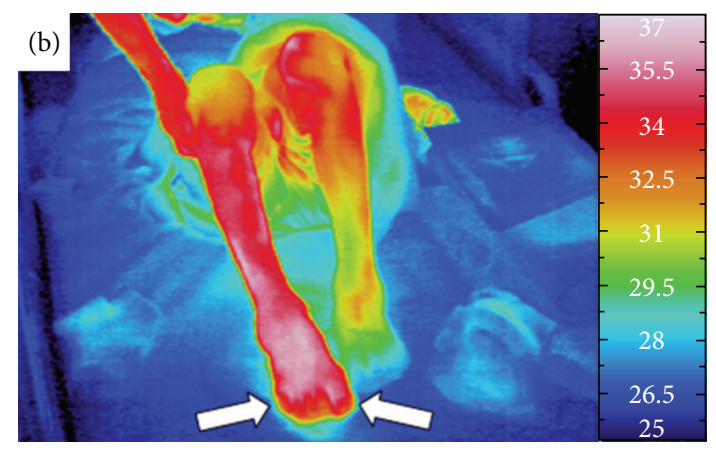

(b)

FIGURE 2: Examples of irregular thermographic patterns of diabetic foot patients with osteomyelitis. (a) Thermographic image of a patient with angiopathy. An increased skin temperature is not seen in any area. Arrow indicates the location of diabetic foot with osteomyelitis. (b) Thermographic image of a patient with inflammation in the soft tissue. The area of increased skin temperature extends to the knee due to cellulitis. Arrows indicate the locating of diabetic foot with osteomyelitis.

was cross-sectional, and the causal relationship between the ankle pattern and osteomyelitis could not be substantiated. A longitudinal study will be needed for investigation of the causal relationship, as well as the effectiveness of this method for prognostic evaluation.

\section{Conclusions}

We investigated thermographic findings in DF patients with and without osteomyelitis and assessed the validity of screening for osteomyelitis using thermography. An area of increased skin temperature extending to the ankle was seen in DF patients with osteomyelitis. Furthermore, this thermographic finding (the ankle pattern) was shown to be quite valid, with high positive predictive value. Thermography might therefore be useful for screening for osteomyelitis in patients with DF.

\section{Acknowledgment}

This work was supported by a Grant from the National Center for the Global Health and Medicine (22-120).

\section{References}

[1] The International Working Group on the Diabetic Foot, The International Consensus on the Management and Prevention of the Diabetic Foot, International Working Group on the Diabetic Foot, Amsterdam, The Netherlands, 2007.

[2] N. Singh, D. G. Armstrong, and B. A. Lipsky, "Preventing foot ulcers in patients with diabetes," Journal of the American Medical Association, vol. 293, no. 2, pp. 217-228, 2005.

[3] R. G. Frykberg, T. Zgonis, D. G. Armstrong et al., "Diabetic foot disorders. A clinical practice guideline (2006 revision)," Journal of Foot and Ankle Surgery, vol. 45, no. 5, pp. S1-S66, 2006.

[4] H. E. Resnick, E. A. Carter, R. Lindsay et al., "Relation of lowerextremity amputation to all-cause and cardiovascular disease mortality in American Indians: the strong heart study," Diabetes Care, vol. 27, no. 6, pp. 1286-1293, 2004.
[5] G. Ragnarson Tennvall and J. Apelqvist, "Health-related quality of life in patients with diabetes mellitus and foot ulcers," Journal of Diabetes and Its Complications, vol. 14, no. 5, pp. 235-241, 2000.

[6] M. H. Nabuurs-Franssen, M. S. P. Huijberts, A. C. Nieuwenhuijzen Kruseman, J. Willems, and N. C. Schaper, "Health-related quality of life of diabetic foot ulcer patients and their caregivers," Diabetologia, vol. 48, no. 9, pp. 1906-1910, 2005.

[7] W. J. Jeffcoate and B. A. Lipsky, "Controversies in diagnosing and managing osteomyelitis of the foot in diabetes," Clinical Infectious Diseases, vol. 39, no. 2, pp. S115-S122, 2004.

[8] A. R. Berendt, E. J. G. Peters, K. Bakker et al., "Diabetic foot osteomyelitis: a progress report on diagnosis and a systematic review of treatment," Diabetes/Metabolism Research and Reviews, vol. 24, no. 1, pp. S145-S161, 2008.

[9] F. Game, "Management of osteomyelitis of the foot in diabetes mellitus," Nature Reviews Endocrinology, vol. 6, no. 1, pp. 43-47, 2010.

[10] M. Oe, R. R. Yotsu, H. Sanada, T. Nagase, and T. Tamaki, "Thermographic findings in a case of type 2 diabetes with foot ulcer and osteomyelitis," Journal of Wound Care, vol. 21, no. 6, pp. 274-278, 2012.

[11] D. G. Armstrong, K. Holtz-Neiderer, C. Wendel, M. J. Mohler, H. R. Kimbriel, and L. A. Lavery, "Skin temperature monitoring reduces the risk for diabetic foot ulceration in high-risk patients," The American Journal of Medicine, vol. 120, no. 12, pp. 1042-1046, 2007.

[12] L. A. Lavery, K. R. Higgins, D. R. Lanctot et al., "Preventing diabetic foot ulcer recurrence in high-risk patients: use of temperature monitoring as a self-assessment tool," Diabetes Care, vol. 30, no. 1, pp. 14-20, 2007.

[13] L. A. Lavery, K. R. Higgins, D. R. Lanctot et al., "Home monitoring of foot skin temperatures to prevent ulceration," Diabetes Care, vol. 27, no. 11, pp. 2642-2647, 2004.

[14] T. Nagase, H. Sanada, K. Takehara et al., "Variations of plantar thermographic patterns in normal controls and non-ulcer diabetic patients: novel classification using angiosome concept," Journal of Plastic, Reconstructive and Aesthetic Surgery, vol. 64, no. 7, pp. 860-866, 2011.

[15] K. Roback, M. Johansson, and A. Starkhammar, "Feasibility of a thermographic method for early detection of foot disorders in 
diabetes," Diabetes Technology and Therapeutics, vol. 11, no. 10, pp. 663-667, 2009.

[16] M. Bharara, J. E. Cobb, and D. J. Claremont, "Thermography and thermometry in the assessment of diabetic neuropathic foot: a case for furthering the role of thermal techniques," International Journal of Lower Extremity Wounds, vol. 5, no. 4, pp. 250-260, 2006.

[17] J. Valabhji, N. Oliver, D. Samarasinghe, T. Mali, R. G. J. Gibbs, and W. M. W. Gedroyc, "Conservative management of diabetic forefoot ulceration complicated by underlying osteomyelitis: the benefits of magnetic resonance imaging," Diabetic Medicine, vol. 26, no. 11, pp. 1127-1134, 2009.

[18] L. Norgren, W. R. Hiatt, J. A. Dormandy, M. R. Nehler, K. A. Harris, and F. G. R. Fowkes, "Inter-society consensus for the management of peripheral arterial disease (TASC II)," Journal of Vascular Surgery, vol. 45, no. 1, pp. S5-S67, 2007.

[19] R. Rubin and D. S. Strayer, Rubin's Pathology: Clinicopathologic Foundations of Medicine, Lippincott Williams \& Wilkins, Baltimore, Md, USA; A Wolters Kluwer Business, Alphen aan den Rijn, The Netherlands, 6th edition, 2012.

[20] D. G. Armstrong, L. A. Lavery, P. J. Liswood, W. F. Todd, and J. A. Tredwell, "Infrared dermal thermometry for the high-risk diabetic foot," Physical Therapy, vol. 77, no. 2, pp. 169-177, 1997.

[21] K. Nishide, T. Nagase, M. Oba et al., "Ultrasonographic and thermographic screening for latent inflammation in diabetic foot callus," Diabetes Research and Clinical Practice, vol. 85, no. 3, pp. 304-309, 2009.

[22] J. L. Cronenwett and K. W. Jonston, Rutherford's Vascular Surgery, Elsevier, Philadelphia, Pa, USA, 7th edition, 2010. 


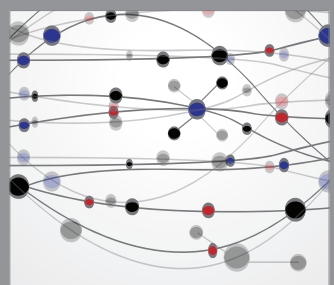

The Scientific World Journal
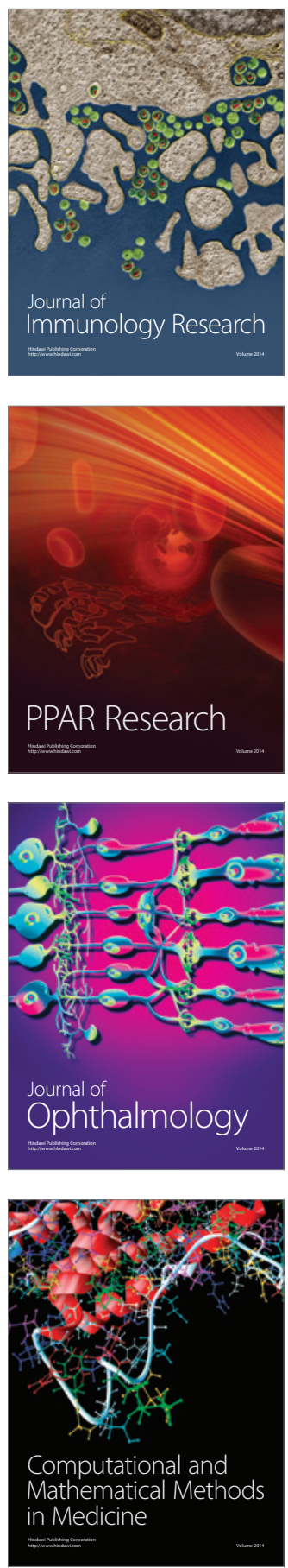

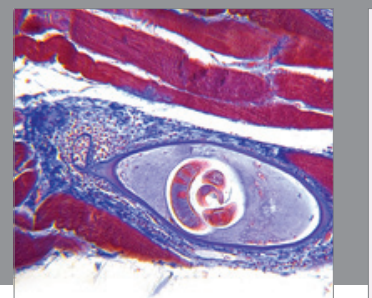

Gastroenterology

Research and Practice
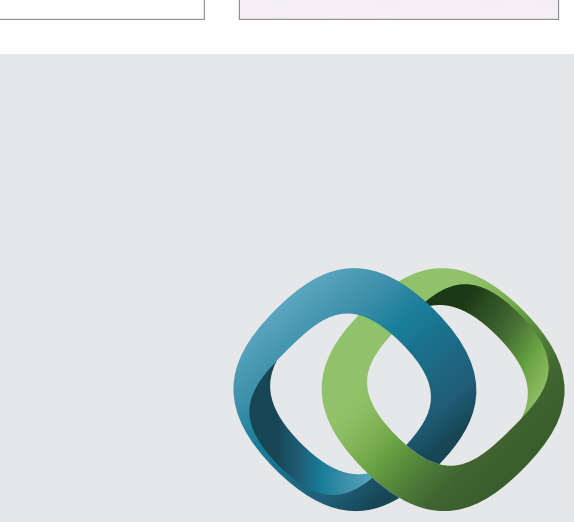

\section{Hindawi}

Submit your manuscripts at

http://www.hindawi.com
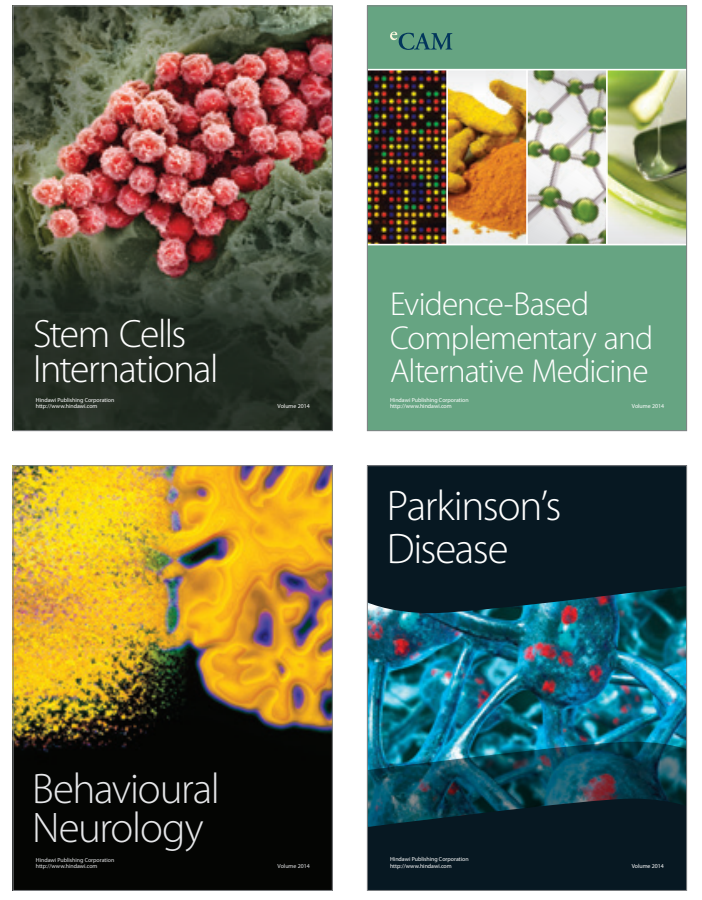
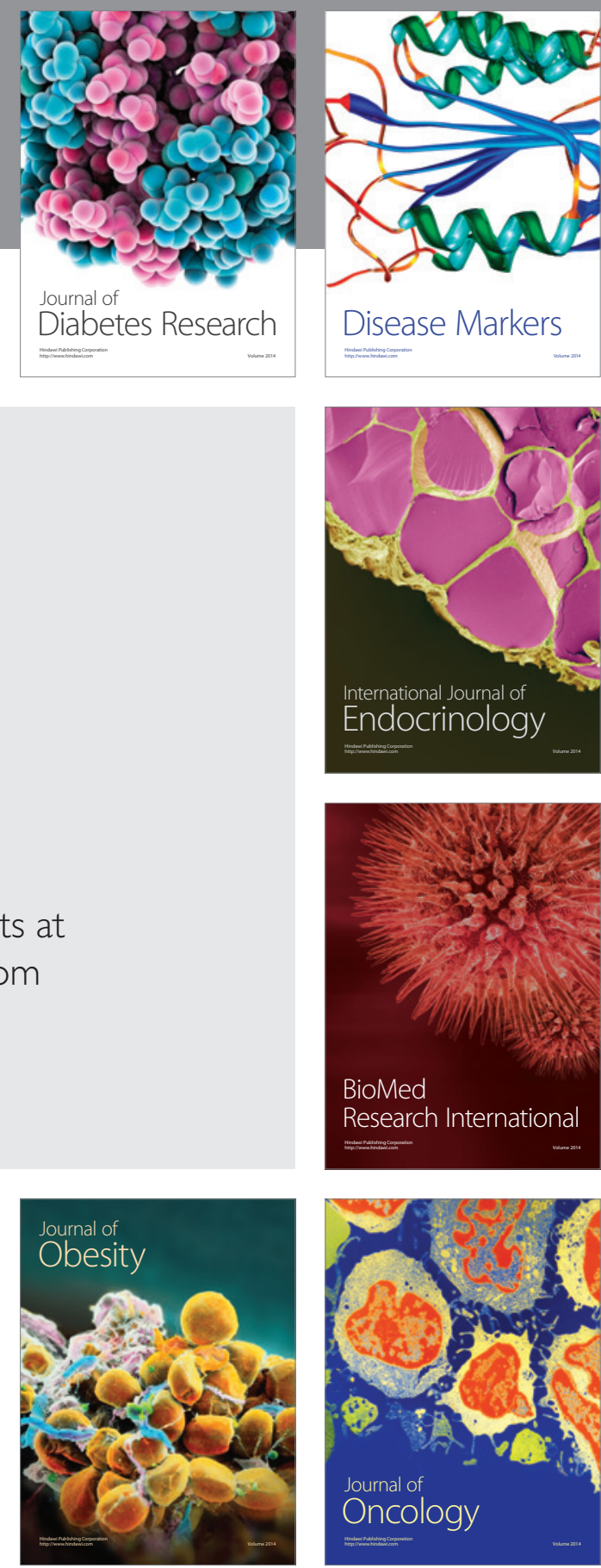

Disease Markers
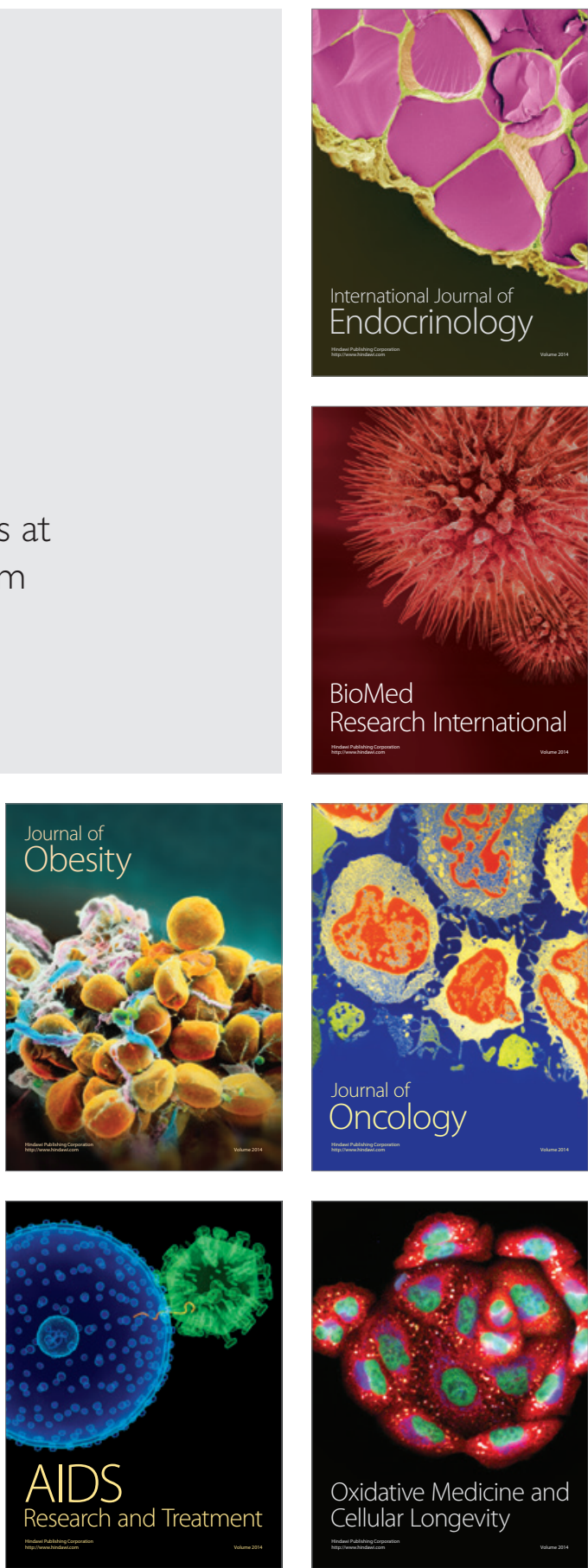